\title{
Revision des Heilmittelgesetzes: Transparenz ist die beste Arznei
}

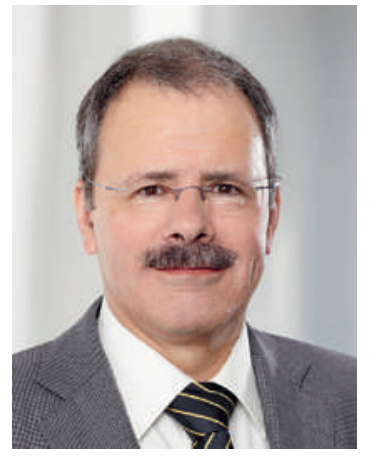

Voraussichtlich in der JuniSession wird der Nationalrat mit der Revision des Heilmittelgesetzes (HMG) beginnen. Die Ziele sind unter anderem: kinderspezifische Arzneimittel zu fördern, die Zulassung vor allem von komplementärmedizinischen Produkten zu erleichtern, die Arzneimittelinformation $\mathrm{zu}$ verbessern sowie Transparenz über so genannte geldwerte Vorteile zu schaffen, das heisst über Boni und Rabatte, welche die Hersteller den bestellenden Arztpraxen, Spitälern und Apotheken gewähren.

Nicht mehr zur Diskussion steht eine Einschränkung oder sogar Abschaffung der Selbstdispensation. Diese ist vorläufig zurückgestellt. Hingegen weiterhin geplant ist - trotz Vorbehalten auch von unserer Seite - eine teilweise rezeptfreie Abgabe von verschreibungspflichtigen Medikamenten durch Apotheken.

\section{Studien für kinderspezifische Arzneimittel und für Arzneimittel bei betagten Menschen sind zu fördern.}

Die FMH beurteilt die vorgesehene HMG-Revision grundsätzlich positiv. Wir begrüssen das Anliegen der verbesserten Patientensicherheit bei der Anwendung von Arzneimitteln. Zentral sind für uns auch Verbesserungen bei der Medikamenteninformation. Die Umstellung auf das elektronische
Arzneimittelkompendium bringt einen Verlust von Informationen. Dieser Informationsverlust kann und muss über das HMG korrigiert werden. Weiter unterstützen wir die vorgesehenen Studien und die Datenbank für kinderspezifische Arzneimittel. Die FMH regt an, eine analoge Regelung für Arzneimittel bei betagten Menschen einzuführen. Bei dieser wachsenden Bevölkerungsgruppe haben die behandelnden Ärztinnen und Ärzte zu wenig durch Studien gesicherte Evidenz.

\section{Revision HMG: FMH unterstützt die} Forderung nach mehr Transparenz.

Einverstanden ist die FMH auch mit der vorgesehenen Offenlegungspflicht für wirtschaftliche Beteiligungen an der Pharma- und Medizinprodukteindustrie. Transparenz ist für uns eine Selbstverständlichkeit. Wer in einem Führungs- oder Aufsichtsgremium sitzt oder eine Beratungs- bzw. Expertenrolle hat, legt dies offen. Und wer beim Einkauf von Heilmitteln Vergünstigungen bekommt, weist dies in der Buchhaltung transparent aus. Intransparenz in diesem sensiblen Bereich kann zu Interessenkonflikten beim sicheren Einsatz von Heilmitteln führen, was in der Folge auch das Vertrauen zwischen Arzt und Patient und damit die Glaubwürdigkeit eines ganzen Berufsstandes beschädigen könnte.

Transparenz ist ebenso nötig bezüglich der Frage, wie mit Vergünstigungen umgegangen werden soll. Hier braucht es eine offene Auslegeordnung der Anteile und des Zusammenspiels sämtlicher Beteiligten: Versicherer, Patienten, Ärzte, Spitäler und Industrie. Die FMH stellt sich dieser Herausforderung.

Dr. med. Jürg Schlup, Präsident der FMH 